\title{
Über den Abbau der Aminosäuren bei der Hefegärung.
}

Von

Otto Neubauer und Konrad Fromherz.

(Aus der II. medizinischen Klinik in München.)

(Der Redaktion zugegangen am 1. November 1910.)

In einer Reihe von Untersuchungen hat F. Ehrlich ${ }^{1}$ ) gezeigt, daß die bei der Hefegärung entstehenden Fuselöle nicht etwa aus dem vergorenen Zucker, sondern aus den der Einwirkung der gärenden Hefe unterliegenden Aminosäuren gebildet werden; und er konnte als ganz allgemein gültiges Gesetz feststellen, daß jede Aminosäure durch gärende Hefe in den Alkohol mit der nächst niederen Zahl von Kohlenstoffatomen übergeführt wird, ein Vorgang, der durch folgende Formel ausgedrückt werden kann:

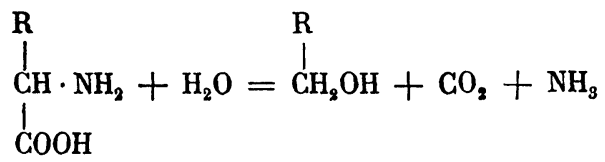

Diese Bruttoformel bringt eine Abspaltung von Ammoniak und Kohlensäure zum Ausdruck, die im ganzen ohne Aufnahme oder Abgabe von Sauerstoff, nur unter Eintritt eines Moleküls Wasser, verläuft, demnach als eine einfache hydrolytische Desaminierung und $\mathrm{CO}_{2}$-Abspaltung erscheint. Dieser Abbau betrifft, wie Ehrlich gezeigt hat, von den beiden optisch aktiven Modifikationen der $\alpha$-Aminosäuren immer nur die im natürlichen Eiweiß vorkommende optisch aktive Modifikation;

1) F. Ehrlich, Zeitschr. d. Ver. d. deutschen Zuckerind., Bd. LV, S. 539 (1905). Bioch. Zeitschr., Bd. II, S. 52 (1906). Ber. d. d. chem. Ges, Bd. XXXIX, S. 4072 (1906). Ebenda, Bd. XL, S. 1027 (1907). Landwirtschaftl. Jahrbücher, Bd. XXXVIII. Erg.-Bd. V, S. 289 (1909). 
bei Zusatz einer racemischen Aminosäure bleibt daher der optische Antipode unangegriffen zurück.

Es ist nun von Interesse, diesen Abbau der Aminosäuren durch die lebende Hefezelle mit den Abbauprozessen zu vergleichen, welche die Aminosäuren im Organismus der Säugetiere erfahren. Durch eine Reihe von Untersuchungen ${ }^{2}$ ) ist festgestellt, daß hier die Aminosäuren über die um ein Kohlenstoffatom niederen Fettsäuren abgebaut werden.

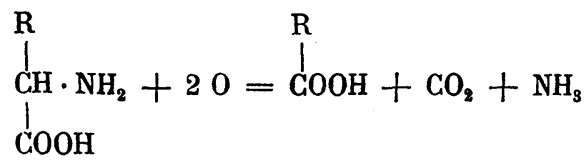

Hier handelt es sich also im ganzen um einen oxydativen Prozeß.

Der eine von uns hat seinerzeit den Nachweis zu erbringen versucht, daß bei diesem $A b b a u$ intermediär die der Aminosäure entsprechende Ketonsäure entsteht, wodurch sich die Formel zunächst in 2 auflösen läßt:

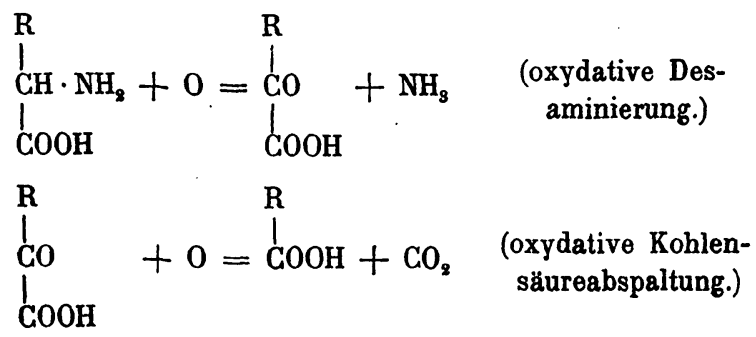

Wir haben uns nun die Frage vorgelegt, ob die Vorgänge bei der Hefezelle und beim höheren Tier von vornherein verschieden verlaufen, oder ob nicht vielleicht wenigstens ein Teil des Weges, den der Abbau einschlägt, beiden gemeinsam ist; von der Beantwortung dieser Frage ließen sich Aufklärungen über die Stoffwechselvorgänge sowohl in der Hefezelle als auch im Säugetierorganismus erwarten.

Die Hauptfrage war die, ob auch bei dem Abbau der Aminosäure zum nächsten niederen Alkohol, wie ihn die Hefezelle leistet, Ketonsäure als Zwischenprodukt auftritt.

1) Literatur bei Otto Neubauer, D. Arch. f. klin. Med., Bd. XCV, S. 120 (1909). 
Um diese Frage bejahen zu dürfen, schien es uns nötig, zwei Dinge nachzuweisen:

1. Daß Aminosäure in Ketonsäure übergehen kann; zu diesem Zwecke war eine Aminosäure gärender Zuckerlösung zuzusetzen und im Gärprodukt die entsprechende Ketonsäure nachzuweisen. Die im Eiweiß vorkommenden Aminosäuren schienen uns zu diesem Zweck von vornherein wenig geeignet, denn die entsprechenden Ketonsäuren mußten, wenn sie als Zwischenprodukte auftraten, eben glatt weiter zersetzlich sein. Wir haben deshalb eine körperfremde Aminosäure benützt, die Phenylaminoessigsäure $\mathrm{C}_{6} \mathrm{H}_{5} \cdot \mathrm{CHNH}_{2} \cdot \mathrm{COOH}$, die dem einen von uns schon zu Untersuchungen beim Säugetier gedient hatte $\left.{ }^{1}\right)$ nach Analogie mit den dort gewonnenen Erfahrungen war anzunehmen, daß die ihr entsprechende Ketonsäure, die Phenylglyoxylsäure $\mathrm{C}_{6} \mathrm{H}_{5} \cdot \mathrm{CO} \cdot \mathrm{COOH}$, gegenüber weiteren $\mathrm{Ab}$ bauvorgängen relativ beständig sein und infolgedessen dem Nachweis sich nicht entziehen würde.

2. Daß $\alpha$-Ketonsäuren bei der Vergärung dasselbe Endprodukt liefern wie die $\alpha$-Aminosäuren, d. i. den nächst niederen Alkohol. Hier bot am meisten Aussicht auf Erfolg die Untersuchung einer Ketonsäure, die einer natürlichen Aminosäure entspricht. Wir wählten die dem Tyrosin entsprechende p-Oxy phenyl-brenztraubensäure $\mathrm{C}_{6} \mathrm{H}_{4} \mathrm{OH} \cdot \mathrm{CH}_{2} \cdot \mathrm{CO} \cdot \mathrm{COOH}$, weil diese Substanz von uns schon zu Untersuchungen im höheren Organismus verwendet worden war, $\left.{ }^{1}\right)$ und weil das zu erwartende Endprodukt, der p-Oxyphenyläthylalkohol $\mathrm{C}_{6} \mathrm{H}_{4} \mathrm{OH} \cdot \mathrm{CH}_{2} \cdot \mathrm{CH}_{2} \mathrm{OH}$, der für den Nachweis sehr günstige Eigenschaften besitzt, von F. Ehrlich als Endprodukt der Tyrosinvergärung bereits nachgewiesen worden ist. ${ }^{2}$ )

Es lag ferner von vornherein im Plane der Untersuchung, auf etwa auftretende Nebenprodukte, speziell auf Alkoholsäuren, zu achten und ihre Rolle bei den Abbauvorgängen aufzuklären.

1) Ot to Neubauer, D. Arch. f. klin. Med., Bd. XGV, S. 231 und 229 (1909). O. Neubauer und W. Groß, Diese Zeitschrift, Bd. LXVII, S. 219 (1910).

2) F. Ehrlich, Ber. d. d. chem. Ges., Bd. XL, S. 1047 (1907). 


\section{Vergärung von Phenylaminoessigsäure.}

F. Ehrlich ${ }^{1}$ ) hat gezeigt, daß von inaktiver Phenylaminoessigsäure der größere Teil der rechtsdrehenden Komponente abgebaut wird und als Endprodukt Benzylalkohol liefert, während der linksdrehende Anteil, vermischt mit einer wechselnden Menge der racemischen Verbindung, unverändert zurückbleibt.

Wir folgten beim Ansetzen der Gärversuche im wesentlichen Ehrlichs Vorschriften. Unsere Versuche sind mit 2 verschiedenen Hefesorten ausgeführt, die in frischem Zustand von der Bäckerinnung München bezogen wurden; die eine Sorte ist als «gewöhnliche (stärkehaltige) Bierhefe» bezeichnet, die andere als «Getreidepreßhefe» von Wieninger, Rittsteig bei Passau. Auf je $10 \mathrm{~g}$ Phenylaminoessigsäure (bezogen von Kahlbaum) verwendeten wir 50-100 g Hefe, 200-350 g Rohrzucker, $3-3^{1 / 2} 1$ Leitungswasser. Die Vergärung war jeweils nach 2-3 Tagen beendet.

Darnach wurde die Hefe abfiltriert und das Filtrat im Vakuum auf etwa 1/10 des Volumens eingeengt; dabei schied sich Phenylaminoessigsäure ab, die abfiltriert und optisch untersucht wurde (in salzsaurer Lösung); übereinstimmend mit den Angaben Ehrlichs erwies sie sich immer als mehr oder weniger stark linksdrehend.

Die Produkte, die mittels a Getreidehefes gewonnen waren, waren meist stärker linksdrehend als die durch «Bierhefe> gebildeten. Z. B.:

Versuch 1. $20 \mathrm{~g}$ Aminosäure, $700 \mathrm{~g}$ Rohrzucker in 61 Wasser mit $100 \mathrm{~g}$ \&Bierhefe, vergoren.

Zurlickgewonnen $11,5 \mathrm{~g}$ Aminosăure $[\alpha] \mathrm{D}=-7,8^{\circ}$.

Versuch 2. $40 \mathrm{~g}$ Aminosăure, $1300 \mathrm{~g}$ Rohrzucker in 141 Wasser, mit $300 \mathrm{~g}$ \&Bierhefe, vergoren.

Zurückgewonnen $24,3 \mathrm{~g}$ Aminosäure $[\alpha] \mathrm{D}=-25,7^{\circ}$.

Versuch 3. $10 \mathrm{~g}$ Aminosäure, $350 \mathrm{~g}$ Rohrzucker in 31 Wasser, mit $100 \mathrm{~g}$ \& Getreidehefe vergoren.

Zurückgewonnen $3,0 \mathrm{~g}$ Aminosäure $[\alpha] \mathrm{D}=-45,0^{\circ}$.

Versuch 4. 17,5 g Aminosäure, $650 \mathrm{~g}$ Rohrzucker in 61 Wasser, mit $150 \mathrm{~g}$ \&Getreidehefe> vergoren.

Zurückgewonnen $8,0 \mathrm{~g}$ Aminosäure $[\alpha] \mathrm{D}=-41,0^{\circ}$.

1) F. Ehrlich, Biochem. Zeitschr., Bd. VIII, S. 438 (1908). 
Durch wiederholtes Vergärenlassen der zurückgewonnenen linksdrehenden Phenylaminoessigsäure gelang es, wie schon Ehrlich angibt, die Linksdrehung immer weiter zu steigern; die höchste Drehung, die wir auf diese Weise erzielten, war $[\alpha]_{D}=-132,2^{\circ}$ (optisch reine l-Phenylaminoessigsäure hat nach Emil Fischer und 0 . Weichhold ${ }^{1}$ ) eine spezifische Drehung $\left.[\alpha] \mathrm{D}=-157,8^{\circ}\right)$.

Beim Einengen der vergorenen Lösung im Vakuum haben wir nie Benzaldehydgeruch wahrnehmen können, außer wenn das Eindampfen bis zur Trockene fortgesetzt wurde; wir vermuten deshalb, daß dieser Benzaldehydgeruch nur infolge künstlicher Zersetzung (der vorhandenen Phenylglyoxylsäure, s. unten S. 331) auftritt.

Das eingeengte Filtrat wurde mit Schwefelsäure angesäuert und im Kutscher-Steudelschen Extraktionsapparat 2 Tage lang mit Äther extrahiert, die ätherische Lösung durcb trockenes Filterpapier filtriert.

Die ätherische Lösung wurde dann wiederholt mit Bisulfitlauge, die vereinigten Bisulfitlösungen mit frischem Äther ausgeschüttelt. Bei diesem Verfahren mußte vorhandene Ketonsäure (Phenylglyoxylsäure) in die Bisulfitlauge übergehen, während andere ätherlösliche Stoffe in Äther gelöst bleiben mußten

A. Aus der Bisulfitlösung wurde nach starkem An. säuern die schweflige Säure durch Erwärmen auf dem Wasser. bade und Durchblasen eines Luftstromes verjagt; dann wurde wieder mit Äther extrahiert und der Rückstand des Äther. extraktes in Wasser aufgenommen, wobei meist noch geringt Mengen Bernsteinsäure abzutrennen waren. Der größte Tel des sirupartigen Extraktes löste sich jedoch in Wasser seh leicht. Die Lösung wurde in der Kälte mit salzsaurem Pheny\} hydrazin versetzt, wodurch sofort ein gelber krystallinische: Niederschlag entstand, der abgesaugt und aus heißem Alkohd und heißem Wasser umkrystallisiert wurde: gelbe lange Nadel vom Schmelzpunkt $160-163^{\circ}$ (unter Aufschäumen und Zer setzung), die bei der Analyse einen $\mathrm{N}$-Wert ergaben, der mit den des Phenylhydrazons der Phenylglyoxylsäure übereinstimmt

1) E. Fis cher und O. Weichhold, Ber. d. d. chem. Ges., Bd. XL, S. 1290 (1908). 
$0,1175 \mathrm{~g}$ Substanz liefern $12,3 \mathrm{ccm} N\left(18,5^{\circ}, 723 \mathrm{~mm}\right)$ entsprechend $11,66 \% \mathrm{~N}$

Berechnet für $\mathrm{C}_{8} \mathrm{H}_{6} \mathrm{O}_{2}: \mathrm{N} \cdot \mathrm{NH} \cdot \mathrm{C}_{6} \mathrm{H}_{5}: 11,67 \%$ » Ausbeuten an umkrystallisiertem Produkt:

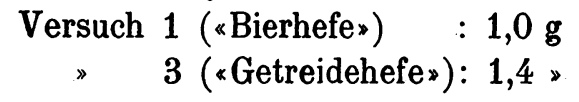

Im ganzen haben wir aus 8 Versuchen $12,9 \mathrm{~g}$ reines Phenylglyoxylsäure-phenylhydrazon erhalten.

Zur Kontrolle haben wir auch 3 Leerversuche (zwei mit 200 resp. $500 \mathrm{~g}$ *Bierhefe , einen mit $150 \mathrm{~g}$ *Getreidehefe» und dem entsprechenden Rohrzuckerzusatz) angesetzt; die «Bisulfitfraktion» lieferte hier mit salzsaurem Phenylhydrazin entweder gar keinen Niederschlag (Versuch mit «Getreidehefe») oder Spuren einer schmierigen Fällung (Versuche mit *Bierhefe»), aber kein krystallisiertes Hydrazon.

Damit ist nachgewiesen, daß auch beim Abbau der Phenylaminoessigsäure durch Hefe Phenylglyoxylsäure $\mathrm{C}_{6} \mathrm{H}_{5} \cdot \mathrm{CO} \cdot \mathrm{COOH}$ in erheblichen Mengen entsteht.

B. Die ätherische Lösung, welche die Substanzen enthielt, die nicht in Bisulfitlauge übergegangen waren, wurde abdestilliert; der Rückstand wurde zur Entfernung der Bernsteinsäure mit Normalnatronlauge neutralisiert, im Vakuum eingedampft und mit Alkohol extrahiert, wobei das Natriumsalz der Bernsteinsäure ungelöst zurückblieb.

Die filtrierte alkoholische Lösung wurde zur Trockene verdampft, mit Natronlauge alkalisch gemacht und mit Äther ausgeschüttelt; in den Äther mußte jetzt der von F. Ehrlich bei der Vergärung von Phenylaminoessigsäure bereits aufgefundene Benzylalkohol übergehen; beim Verdampfen des ätherischen Extraktes erhielten wir in der Tat einen in Benzol löslichen flüssigen, neutral reagierenden Rückstand, der offenbar als Benzylalkohol $\mathrm{C}_{6} \mathrm{H}_{5} \cdot \mathrm{CH}_{2} \mathrm{OH}$ anzusprechen war.

Die ausgeätherte alkalische Lösung wurde mit Kohlensäure gesättigt und dann neuerdings mit Äther extrahiert; die Ätherrückstände aus den verschiedenen Versuchen wurden vereinigt; sie krystallisierten in langen Prismen, die aus Benzol- 
Ligroin einmal umkrystallisiert, bei $92^{\circ}$ schmolzen; die Ausbeute aus 4 Versuchen (entspr. $600 \mathrm{~g}$ Hefe) betrug 0,45 g. Eine Substanz von denselben Eigenschaften konnte auch in den entsprechenden Fraktionen der Leerversuche nachgewiesen werden. Nach dem Schmelzpunkt und den übrigen Eigenschaften (Millonsche Reaktion) lag der schon von F. Ehr-

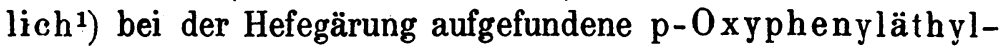
alkohol $\mathrm{C}_{6} \mathrm{H}_{4} \mathrm{OH} \cdot \mathrm{CH}_{2} \cdot \mathrm{CH}_{2} \mathrm{OH}$ vor, der dem Tyrosin des Hefeeiweißes entstammt.

Zur Gewinnung der sauren Bestandteile des Ätherextraktes wurde schließlich die bicarbonatalkalische wässerige Lösung mit Salzsäure angesäuert und nochmals ausgeäthert; der Äther wurde entwässert, abdestilliert, der sirupöse Rückstand im Vakuum über Schwefelsäure getrocknet.

Bei einem Teil der mit «Bierhefe» angesetzten Versuche schieden sich dabei über Nacht dachförmig abgeschnittene zu Rosetten angeordnete Prismen ab, die, als sie sich nicht mehr vermehrten, abfiltriert und mit kaltem Wasser gewaschen wurden (Menge bei Versuch 2: 0,2 g). Das Filtrat wurde wieder im Vakuum eingetrocknet und dann mit Benzol ausgekocht; ebenso wurde mit den nicht in dieser Weise krystallisierenden Sirupen aus den anderen Versuchen verfahren. Dabei blieb ein unvollständig krystallisierter ungelöster Rest, der aus heißem Wasser umkrystallisiert ebenfalls die beschriebenen Prismen lieferte (aus 3 Versuchen mit «Getreidehefe zusammen 0,2 bis $0,3 \mathrm{~g}$ ); ein weiterer Teil krystallisierte bei den «Bierhefe»-Versuchen beim Erkalten der Benzollösung in Nadeln aus. Diese Fraktionen, die in ihren Eigenschaften übereinstimmten, wurden nach dem Umkrystallisieren aus Wasser zusammen weiter verarbeitet.

Nach mehrfachem Umkrystallisieren aus wenig heißem Wasser wurde die Substanz in weißen, spröden, dachförmig endenden Prismen erhalten, die in heißem Wasser löslich waren, schwerer in kaltem, sehr schwer in Benzol. Sie gaben eine stark positive Lassaignesche Stickstoffprobe, schmolzen bei 190-191 ${ }^{\circ}$ (korr.) scharf ohne Zersetzung, hatten saure Eigenschaften und drehten stark nach links.

1) F. Ehrlich, Landwirtschaftl. Jahrb. 23, Erg.-Bd. V, S. 307 (1909). 
In allen ihren Eigenschaften, auch in ihrem Schmelzpunkt stimmt die Substanz mit der d-Acetyl-Phenylaminoessigsäure überein, die der eine von uns in Gemeinschaft mit 0 . Warburg ${ }^{1}$ ) bei Zusatz von Phenylaminoessigsäure zur künstlich durchbluteten Hundeleber erhalten hat; nur die optische Drehung ist zwar ebenso stark, geht aber in ent ge geng e s etz ter Richtung.

$0,1348 \mathrm{~g}$ in $24,5655 \mathrm{~g}$ wässeriger Lösung drehen bei $20^{\circ}$ die Ebene des polarisierten Natriumlichtes im $2 \mathrm{dm}$ langen Rohr 2,1440 nach links; daraus berechnet:

$$
[\alpha]_{D}^{200}=-195,4^{\circ} \text {. }
$$

Zur Sicherstellung wurde die Substanz titriert und einer Stickstoff bestimmung unterworfen.

Titration: 0,1148 g Substanz verbrauchen (Indikator: Phenolphthalein)

Berechnet für $\mathrm{C}_{9} \mathrm{H}_{10} \mathrm{NO} \cdot \mathrm{COOH}: 5,95$ » »

N-Bestimmung. Dieselbe Menge Substanz, zur N-Bestimmung nach Kjeldahl verwendet, verbraucht $6,10 \mathrm{ccm}$ n/10-Säure, entsprechend $7,26 \% \mathrm{~N}$

Berechnet für $\mathrm{C}_{10} \mathrm{H}_{11} \mathrm{NO}_{3}: 7,44 \%$ »

Damit ist die Bildung von l-Acetyl-Phenylaminoessigsäure bei der Hefegärung der Phenylaminoessigsäure festgestellt. Bei den Leerversuchen wurde sie nicht gebildet; die entsprechenden Fraktionen krystallisierten nicht und erwiesen sich bei der Lassaigneschen Probe als frei von Stickstoff.

Bei den Versuchen mit "Getreidehefe * krystallisierten aus den oben erwähnten Benzollösungen beim Erkalten nicht die Prismen der Acetylverbindung, sondern glänzende Blättchen, die in Wasser sehr leicht löslich waren und von denen verschiedene Präparate Schmelzpunkte von $115-130^{\circ}$ zeigten; beim Einengen der Benzollösungen konnten weitere Mengen dieser Substanz gewonnen werden. Bei den Versuchen mit Bierhefe dagegen krystallisierten hauptsächlich die Prismen der Acetylverbindung neben geringen Mengen der Blättchen, die nicht isoliert werden konnten.

1) 0. Neubauer und O. Warburg, Diese Zeitschrift, Bd. LXX, S. 1 (1910).

Hoppe-Seyler's Zeitschrift f. physiol. Chemie. LXX. 
Ausbeuten an Rohprodukt: Bei 3 Versuchen mit «Getreidehefe * wurden $0,8 \mathrm{~g}$ vom Schmelzpunkt $120^{\circ}$ direkt erhalten. Beim Einengen weitere $0,9 \mathrm{~g}$ vom Schmelzpunkt $115^{\circ}$. Aus 2 anderen Versuchen erhielten wir zusammen $0,9 \mathrm{~g}$ eines aus Benzol umkrystallisierten Produkts vom Schmelzpunkt $121^{\circ}$.

Durch mehrmaliges Umkrystallisieren aus Benzol, teilweise unter Zusatz von wenig Tierkohle, konnte der Schmelzpunkt auf $122-133^{\circ}$, die spezifische Drehung auf $-138,7$ bis $-141,9^{\circ}$ gebracht werden. Nachdem die Substanz nochmals aus wenig Wasser umkrystallisiert war, stieg der Schmelzpunkt auf $133^{\circ}$, die Drehung auf $-151,0^{\circ}$.

$0,0223 \mathrm{~g}$ Substanz gelöst in 1,8975 g Lösung drehen im 1-Dezimeterrohr $1,7^{\circ}$ nach links; spezifisches Gewicht 1,004 .

Daraus berechnet:

$$
[\alpha]_{\mathrm{D}}^{2 \mathrm{~N}^{\circ}}=-150,95^{\circ} \text {. }
$$

$0,1503 \mathrm{~g}$ Substanz gaben $0,3493 \mathrm{~g} \mathrm{CO}_{2}$ und $0,0764 \mathrm{~g} \mathrm{H}_{2} \mathrm{O}$; gefunden: $\quad \mathrm{C}=63,38 \% \quad \mathrm{H}=5,64 \%$ berechnet für $\mathrm{C}_{8} \mathrm{H}_{8} \mathrm{O}_{3}: \quad \mathrm{C}=63,12 \% \quad \mathrm{H}=5,32 \%$.

Analyse, Schmelzpunkt und Drehung, sowie die übrigen Eigenschaften stimmen also für l-Mandelsäure, die der eine von uns als Umwandlungsprodukt der Phenylaminoessigsäure im Organismus des Menschen und Hundes bereits nachgewiesen hat, und die auch bei den Durchblutungen in der Leber entsteht. ${ }^{1}$ )

Es ist somit der Nachweis geführt, daß bei dem Gärungsversuch Phenylaminoessigsäure neben Benzylalkohol auch Phenylglyoxylsäure, l-Mandelsäure und l-Acetylphenylaminoessigsäure liefert.

Die Methode der Isolierung dieser Substanzen sei der Übersicht halber durch folgende Tabelle (s. nächste Seite) nochmals veranschaulicht.

In welchen genetischen Beziehungen stehen die gefundenen Umwandlungsprodukte der Phenylaminoessigsäure zu einander?

1) O. Neubauer und H. Fischer, Diese Zeitschrift, Bd. LXVII, S. 237 (1910). 
Über den Abbau der Aminosäuren bei der Hefegärung. 335

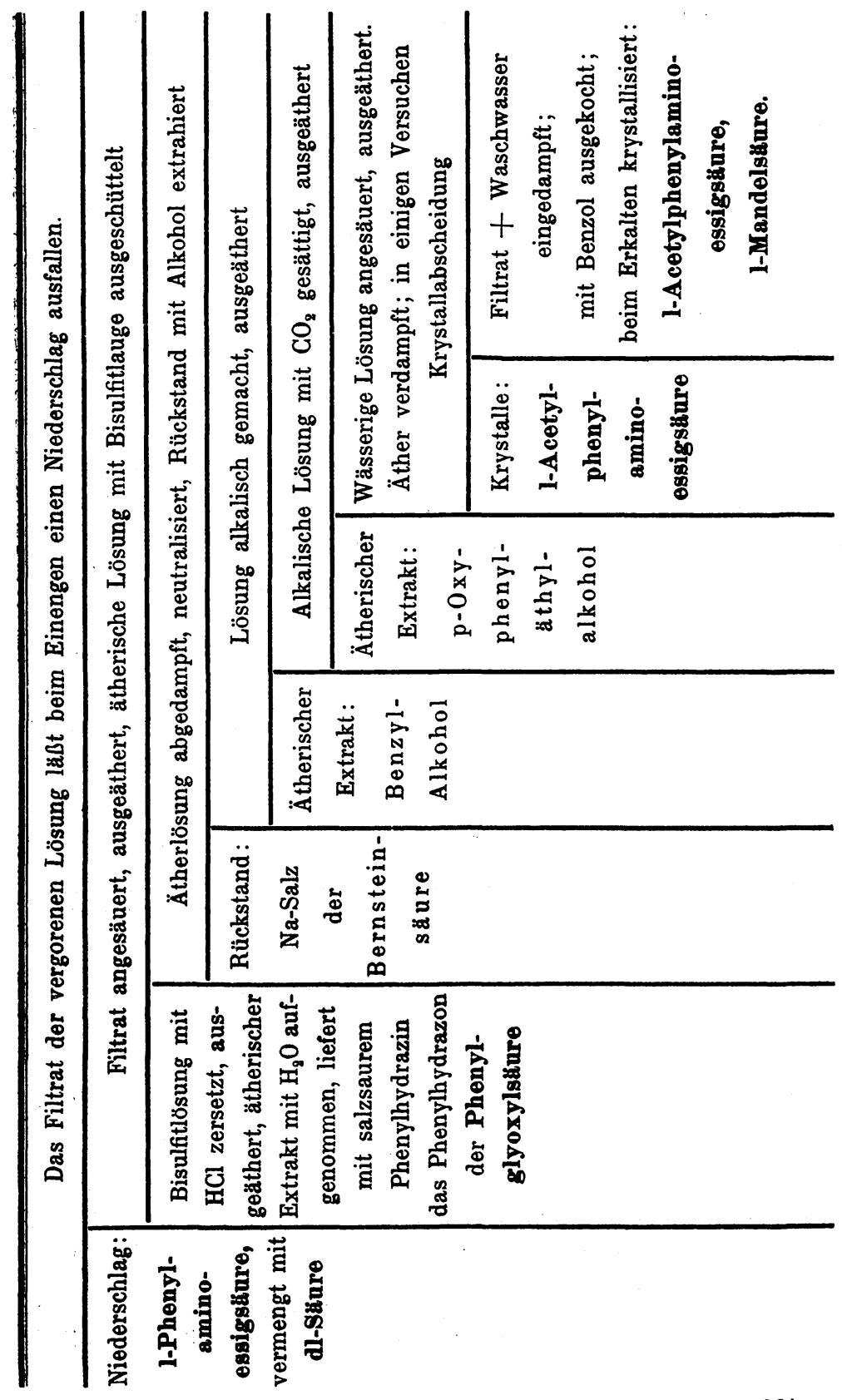


Zunächst ist zu entscheiden, ob aus der Aminosäure zuerst die 1-Mandelsäure und aus dieser durch Oxydation die Phenylglyoxylsäure entstanden ist, oder ob umgekehrt die Mandelsäure als ein sekundäres Reduktionsprodukt der Phenylglyoxylsäure zu deuten ist.

Die erste Annahme ist schon deshalb unwahrscheinlich, weil die l-Mandelsäure wahrscheinlich dem l-Anteil der Phenylaminoessigsäure entspricht, ${ }^{1}$ ) bei der Vergärung aber gerade der d-Anteil abgebaut wird; im gleichen Sinne spricht die Analogie mit dem Verhalten beim Säugetier, ${ }^{1}$ ) resp. in der künstlich durchbluteten Leber. ${ }^{2}$ )

Zur Klarstellung haben wir Vergärungsversuche mit Phenylglyoxylsäure und Mandelsäure (linksdrehender und racemischer) ausgeführt. 7,5 g Phenylglyoxylsäure (resp. Mandelsäure), mit Natronlauge neutralisiert, werden mit $125 \mathrm{~g} \mathrm{Ge}$ treidehefe und $250 \mathrm{~g}$ Rohrzucker in $\left.2^{1}\right|_{2}$ l Leitungswasser gelöst und bei Zimmertemperatur stehen gelassen.

Nach dem Abschluß der Gärung werden die Lösungen wie oben verarbeitet.

Der Gärversuch mit Phenylglyoxylsäure lieferte dabei neben viel unveränderter Substanz ein stark linksdrehendes, ätherisches Extrakt, aus dem 1-Mandelsäure isoliert werden konnte $\left(\alpha_{D}=-150^{\circ}\right)$. Nach der Stärke der Linksdrehung waren ca. $0,19 \mathrm{~g}$ Mandelsäure entstanden. Acetyl-Phenylaminoessigsäure wurde nicht gefunden (der Versuch war mit * Getreidehefe» angestellt worden, s. oben S. 332).

Im Versuch mit Links-Mandelsäure war nur eine ganz geringe Menge von Ketonsäure gebildet worden; es wurden im ganzen $0,023 \mathrm{~g}$ Phenylhydrazon der Phenylglyoxylsäure (Schmelzpunkt nach Umkrystallisieren 1640) erhalten, entsprechend $0,016 \mathrm{~g}$ freier Säure. Dagegen wurde sehr viel Links-Mandelsäure zurückgewonnen.

Auch aus der racemischen Mandelsäure war nur eine sehr kleine Quantität Ketonsäure entstanden; die Ausbeute an

1) O. Neubauer, D. Arch. f. klin. Med., Bd. XCV, S. 237 (1909).

2) O. Neubauer und H. Fischer, Diese Zeitschrift, :Bd. LXVII, S. 237 (1910). 
Über den Abbau der Aminosäuren bei der Hefegärung.

Phenylglyoxylsäure-Phenylhydrazon betrug hier $0,068 \mathrm{~g}$, entsprechend $0,048 \mathrm{~g}$ Ketonsäure. Der Schmelzpunkt des umkrystallisierten Phenylhydrazons lag bei $166^{\circ}$. Die in großer Menge zurückgewonnene Mandelsäure erwies sich als schwach rechtsdrehend (Drehung der in $100 \mathrm{ccm}$ Wasser gelösten, neutralisierten Substanz im $2 \mathrm{dm}-\mathrm{Rohr}+0,34^{\circ}$, entsprechend $0,15 \mathrm{~g}$ d-Mandelsäure). Ein zweiter Versuch ergab ein übereinstimmendes Resultat.

Aus diesen Versuchen ergibt sich also:

1. Daß Phenylglyoxylsäure durch gärende Hefe eine teilweise Reduktion zu l-Mandelsäure erfährt, analog dem Verhalten im Körper des lebenden Hundes und in der künstlich durchbluteten Leber. Die Reduktion betrifft nur einen kleinen Teil der Phenylglyoxylsäure, ist aber geeignet, das Auftreten der relativ geringen Quantität von l-Mandelsäure neben größeren Mengen Phenylglyoxylsäure bei der Vergärung der Phenylaminoessigsäure zu erklären.

2. Daß die Hefe zwar auch imstande ist, Mandelsäure zu Phenylglyoxylsäure zu oxydieren, daß aber dieser Prozeß in so geringem Ausmaße erfolgt, daß er für die Bildung der großen Menge Phenylglyoxylsäure, die bei der Gärung aus der Aminosäure entsteht, kaum in Betracht kommt.

Somit spricht der Ausfall dieser Versuche dafür, daß bei der Vergärung der Aminosäure zunächst die Ketonsäure entsteht, die dann sekundär durch optisch aktive Reduktion teilweise in die aktive Alkoholsäure (Mandelsäure) übergeht.

In demselben Sinne, da $\beta$ die Ketonsäure nicht aus der Alkoholsäure entsteht, spricht ferner der Ausfall der weiter unten (S. 345) angeführten Gärversuche mit p-Oxyphenylbrenztraubensäure und p-Oxyphenylmilchsäure, die beweisen, daß die Ketonsäure bei der Vergärung dasselbe Endprodukt liefert wie die entsprechende Aminosäure, die Alkoholsäure dagegen nicht.

Bei der Bildung der Phenylglyoxylsäure aus der Phenylaminoessigsäure handelt es sich um zwei Prozesse: Ammoniakabspaltung und Oxydation (oxydative Desaminierung). 


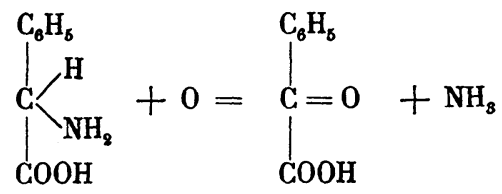

Will man nun nicht annehmen, daß diese beiden Vorgänge gleichzeitig in unbedingter gegenseitiger Abhängigkeit verlaufen (für welche Annahme gar kein Grund vorliegt), so wird man die Frage aufwerfen müssen, welcher von beiden Prozessen dem anderen vorangeht. Die Antwort ergibt sich aus den angeführten Versuchen. Wäre die $\mathrm{NH}_{3}$-Abspaltung der erste Vorgang (der dann natürlich unter Aufnahme von $\mathrm{H}_{2} \mathrm{O}$ erfolgen müßte), so würde als erstes Zwischenprodukt die Alkoholsäure (Mandelsäure) auftreten, eine Annahme, die wir soeben abgelehnt haben. Es bleibt also gar keine einfache Vorstellungsmöglichkeit, als die, die Oxydation(Hydroxylierung) als ersten, die $\mathrm{NH}_{3}$-Abspaltung(Desaminierung) als zweiten Prozeß anzusprechen.

I.<smiles>CCCCC(N)(O)C(=O)O</smiles>

II.<smiles>CCCC(N)(O)C(=O)O</smiles>

Ketonsäure.

Es ist ersichtlich, daß dabei das Hydrat der Iminosäure (vielleicht auch die Iminosäure $\mathrm{C}_{6} \mathrm{H}_{5} \cdot \mathrm{C}(\mathrm{NH}) \cdot \mathrm{COOH}$ selbst) als intermediäres Produkt auftreten muß. ${ }^{1}$ ) Es ist bekannt, daß. Iminosäuren im Reagenzglase sehr leicht in Ketonsäuren übergehen; der Nachweis, daß auch Hefe imstande ist, diese Umwandlung zu bewirken, soll an der bereits bekannten Phenyliminoessigsäure noch geführt werden.

Die gleichen Überlegungen gelten auch für die Entstehung der Ketonsäuren aus Aminosäuren beim Säugetier.

Über die Art der Entstehung der 1-Acetyl-Phenylaminoessigsäure können wir keine bestimmten Angaben machen; ein Anhaltspunkt, um ihr eine wesentliche Rolle als

1) Auch Kno op [Diese Zeitschrift, Bd. LXVII, S. 498 (1910)] macht darauf aufmerksam, daß derartige Substanzen vielleicht beim oxydativen Abbau der Aminosäuren entstehen. 
Zwischenprodukt zuzuweisen, ist gegenwärtig nicht gegeben; doch wird diese Frage noch weiter zu prüfen sein. Es sei auf die Untersuchungen von Knoo ${ }^{1}$ ) hingewiesen, welche zeigen, daß eine Ketonsäure im Tierkörper in die aktive Acetylaminosäure übergehen kann. Sehr merkwürdig ist es, daß die bei der Hefegärung gebildete Acetylverbindung der optische Antipode des bei der Leberdurchblutung entstehenden Pruduktes ist; diese Tatsache spricht jedenfalls nicht dafür, daß die Acetylverbindung aus der Mandelsäure entsteht, denn diese zeigte bei den Gärungsversuchen wie bei den Durchblutungsversuchen die gleiche Drehungsrichtung (links).

\section{Vergärung von p-0xyphenylbrenztraubensäure.}

Diese Versuche sollten eine weitere Stütze für die Annahme bringen, daß die Ketonsäure beim Abbau der Aminosäuren durch gärende Hefe als intermediäre Produkte auftreten, nämlich den Nachweis, daß sie bei der Vergärung dasselbe Endprodukt wie die Aminosäuren liefern: den um ein Kohlenstoffatom ärmeren Alkohol.

Wir haben für diesen Versuch die dem Tyrosin entsprechende Ketonsäure, die p-Oxyphenylbrenztraubensäure

$$
\mathrm{C}_{6} \mathrm{H}_{4} \mathrm{OH} \cdot \mathrm{CH}_{2} \cdot \mathrm{CO} \cdot \mathrm{COOH}
$$

gewählt, welche beim Vergärungsversuch ebenso wie Tyrosin in p-Oxyphenyläthylalkohol $\mathrm{C}_{5} \mathrm{H}_{5} \mathrm{OH} \cdot \mathrm{CH}_{2} \cdot \mathrm{CH}_{2} \mathrm{OH}$ übergehen mußte. Da diese Substanz in einer gewissen Menge aber auch bei den Leerversuchen entsteht, ${ }^{8}$ ) so waren genaue Parallelversuche ohne Zusatz von Ketonsäure erforderlich und die Versuche konnten nur dann beweisend sein, wenn sie bedeutend größere Mengen des Alkohols ergaben als die Leerversuche.

Die Darstellung der p-0xyphenylbrenztraubensäure machte bis jetzt immer Schwierigkeiten, da die erhaltenen Rohprodukte nur schwer und unter großen Verlusten gereinigt und auf den richtigen Schmelzpunkt gebracht werden konnten. Es erwies sich folgende abgeänderte Arbeitsmethode als zweckmäßig:

Je $10 \mathrm{~g}$ umkrystallisierte $\mathrm{p}-0 \mathrm{xy}$ - $\alpha$-benzoylaminozimmtsäure, dargestellt

1) Knoop, Diese Zeitschrift, Bd. LXVII, S. 497 (1910).

2) F. Ehrlich, s. auch oben S. 332. 
nach Vorschrift von Erlenmeyer und Halsey, ${ }^{1}$ ) wurden mit $100 \mathrm{ccm}$ Natronlauge vom spez. Gew. 1,333 übergossen und (im Kjeldahl-Kolben auf dem Drahtnetz) eine halbe Stunde gekocht. Die Substanz löst sich zunächst mit gelbbräunlicher Farbe klar auf, dann scheidet sich unter Ammoniakentwicklung ein dicker geballter Niederschlag von benzoesaurem Natron aus. Es wird jeweils so viel Wasser zugefügt, daß die Masse als Suspension erhalten bleibt und völliges Erstarren vermieden wird, jedoch nicht mehr. Nach einer halben Stunde wird heiß in eine Schale gegossen und der Kolben mit wenig Wasser nachgespült. Nach dem Erkalten wird unter Zerdrücken der Masse mit einem Mörserpistill abgesaugt und mit wenig konzentrierter Natronlauge nachgewaschen, dann scharf abgepreßt. Das Filtrat wird unter Vermeiden einer Erwärmung über $30^{\circ}$ (Kühlung) mit konzentrierter Salzsäure angesäuert, wobei schließlich ein feiner grauer Niederschlag entsteht (Fraktion 1).

Dieser Niederschlag wird nach einer halben Stunde abfiltriert. Er schmilzt getrocknet bei $185-193^{\circ}$; es gelang nicht, daraus Oxyphenylbrenztraubensäure rein darzustellen, und er wurde deshalb nicht weiter verarbeitet.

Das Filtrat von diesem Niederschlag wird $3 \mathrm{mal}$ mit viel Äther ausgeschüttelt, die ätherische Lösung mit wenig konzentrierter Bisulfitlauge (auf je $10 \mathrm{~g}$ Oxybenzoylaminozimmtsäure $20-25 \mathrm{ccm}$ Bisulfitlauge) durchgeschüttelt. Nach einigem Schütteln scheiden sich aus der Bisulfitlösung Krystalle $a b$, und schließlich erstarrt die ganze Lauge zu einem dicken Krystallbrei. Dieser wird nach etwa halbstündigem Stehen ohne Verdünnung abgesaugt und mit wenig kaltem Wasser nachgewaschen. Aus der abfiltrierten Bisulfitlösung gelingt es nicht mehr, nennenswerte Mengen reiner $\mathrm{p}-0 x y$ phenylbrenztraubensäure darzustellen; dagegen besteht die ahgeschiedene weiße Krystallmasse aus der so gut wie reinen Bisulfitverbindung der Ketonsäure; sie wird in Wasser aufgeschwemmt und mit konzentrierter Salzsäure bis zur kongosauren Reaktion versetzt, wobei Lösung eintritt. Darauf wird die schweflige Säure auf dem Wasserbad durch einen Luftstrom verjagt (wobei man bisweilen zweckmäßig nachkontrolliert, ob genügend Säure zugesetzt war). Schon in der Hitze scheidet sich dabei die p-Oxyphęnylbrenztraubensäure in schneeweißen Blättchen $a b$, die sich beim Erkalten noch vermehren. Nach Abfiltrieren, Waschen und Trocknen erhält man so ein Präparat, das bei $213^{\circ}$ (korr. $217^{\circ}$ ) unter mäBiger Gasentwicklung schmilzt, das einheitlich in rhombenförmigen oder sechsseitigen Tafeln krystallisiert ist. Durch Umkrystallisieren aus heißem Wasser ändert sich der Schmelzpunkt nicht mehr. Durch Eindampfen der Mutterlauge erhält man noch eine geringe weitere Krystallisation. Ausbeute aus $70 \mathrm{~g}$ 0xybenzoylaminozimmtsäure: 12,2 g umkrystallisierte 0xyphenylbrenztraubensäure, außerdem eine Nach-

1) Erlenmeyer und Halsey, Annalen, Bd. GCGVII, S. 138. 
krystallisation von $1,5 \mathrm{~g}$ vom Schmelzpunkt $212^{\circ}$ (korr.), das ist $34-38 \%$ der Theorie. ${ }^{1}$ )

1) Nebenprodukte bei der Darstellung der p-Oxyphenylbrenztraubensäure: Die Ursache der Schwierigkeiten, nach der alten Methode die Ketonsäure darzustellen, liegt in der Entstehung von Nebenprodukten bei der Verseifung der p-Oxybenzoylaminozimmtsäure. Von diesen konnte wenigstens eines in reinem Zustand abgetrennt werden; Konstitution und Bildungsweise der Substanz sind jedoch noch nicht vollkommen geklärt.

Überläßt man nach dem Verseifen der p-Oxybenzoylaminozimmtsäure, Abtrennen des benzoesauren Natrons und Ansäuren das Filtrat sich selbst, dann erhält man innerhalb 3 Tagen verschiedene Krystallfraktionen, die wir getrennt der fraktionierten Krystallisation aus Wasser unterwarfen. Auf diese Weise kann man unter Kontrolle des Mikroskops einerseits die Tafeln der p-Oxyphenylbrenztraubensäure rein erhalten, daneben einen in ziemlich derben vierseitigen, abgedachten Prismen krystallisierenden Körper abtrennen und von Gemischen dieser Substanz mit dritten Krystallformen isolieren.

Die Prismen (Substanz B, Ausbeute 5-10\% des Ausgangsmaterials) sind stickstofffrei, geben eine starke Millonsche Reaktion, mit Eisenchlorid eine verschwindende Grünfärbung. Kupferoxyd wird in alkalischer Lösung in Lösung gehalten, jedoch auch beim Kochen nicht reduziert. Mit salzsaurem Phenylhydrazin gibt eine Lösung der Substanz keinen Niederschlag; es schieden sich bei einigem Stehen nur wieder die unveränderten Prismen ab. Beim Kochen mit starker Natronlauge oder Schwefelsäure entsteht p-Oxyphenylbrenztraubensäure (Nachweis durch das Phenylhydrazon). Beim Erhitzen tritt Abspaltung von Wasser, später von Kohlensäure ein; dementsprechend zeigte die Substanz B keinen konstanten Schmelzpunkt.

Im Vakuum über Schwefelsäure oder Phosphorsäureanhydrid verliert der Körper schon bei gewöhnlicher Temperatur, rascher bei $57-100^{\circ}$, Krystallwasser unter Zerfall zu einem amorphen Pulver:

$$
\begin{array}{r}
0,6530 \mathrm{~g} \text { Substanz verloren } 0,0630 \mathrm{~g}: \\
\mathrm{C}_{\mathbf{1 8}} \mathrm{H}_{14} \mathrm{O}_{7}+2 \mathrm{H}_{2} \mathrm{O} \text { ber.: } \mathrm{H}_{2} \mathrm{O}=9,53 \% \\
\text { gef.: } \mathrm{H}_{\mathbf{2}} \mathrm{O}=9,64 \% .
\end{array}
$$
$\mathrm{n} / \mathbf{1 0}-\mathrm{NaOH}$ :

0,1082 g Substanz verbrauchen gegen Phenolphthalein titriert $5,4 \mathrm{ccm}$

Molekulargewicht, 2 basische Säure angenommen: gef. : 400,6

$$
\mathrm{C}_{18} \mathrm{H}_{14} \mathrm{O}_{7}+2 \mathrm{H}_{2} \mathrm{O} \text { ber.: } 378,2 \text {. }
$$

$0,1403 \mathrm{~g}$ Substanz: $0,2970 \mathrm{~g} \mathrm{CO}_{2}$ und $0,0683 \mathrm{~g} \mathrm{H}_{8} \mathrm{O}$.

$\mathrm{C}_{18} \mathrm{H}_{14} \mathrm{O}_{7}+2 \mathrm{H}_{8} \mathrm{O}$ ber.: $\mathrm{G}=57,11 \%, \mathrm{H}=4,81 \%$,

$$
\text { gef. : } \mathrm{C}=57,73 \%, \mathrm{H}=\tilde{5}, 41 \% \text {. }
$$

Die Abweichungen von der Theorie sind durch eine geringe Kohlen- 
Analyse: $0,1447 \mathrm{~g}$ Substanz geben 0,3182 $\mathrm{g} \mathrm{CO}_{2}$ und $0,0604 \mathrm{~g}_{2} \mathrm{O}$.

$$
\mathrm{C}=60,00 \% \quad \mathrm{H}=4,50 \%
$$

Für $\mathrm{C}_{9} \mathrm{H}_{8} \mathrm{O}_{4}$ berechnet: $\mathrm{C}=60,06 \% \quad \mathrm{H}=4,68 \%$.

Phenylhydrazon der p-0xyphenylbrenztraubensäure.

Kalte Lösungen von berechneten Mengen salzsaurem Phenylhydrazin und p-Oxyphenylbrenztraubensäure geben bei der Mischung einen hellgelben krystallinischen Niederschlag, der abfiltriert, in heißem Alkohol gelöst, und bis zur leichten Trübung mit heißem Wasser versetzt, sehr charakteristisch krystallisiert: gelbe kolbenförmige Krystalle, die zu rechtund spitzwinkelig verzweigten, farnkrautblätterähnlichen Gebilden vereinigt sind; bisweilen zeigen die einzelnen Krystalle auch prismatische Form, mit zugespitzten Enden und meist gesägten Kanten; ein charakteristisches Kennzeichen ist jedoch die farnkrautblätterähnliche Anordnung. Schmelzpunkt $167-169^{\circ}$ bei raschestem Erhitzen, bei langsamerem Erhitzen 159-1610, dabei stürmische Zersetzung.

Die lufttrockene Substanz ist im Vakuum über Schwefelsäure und Phosphorsäureanhydrid gewichtskonstant.

Analyse: 0,1794 g Substanz geben $17,4 \mathrm{ccm} \mathrm{N}$ bei $23,5^{\circ}$ und $713 \mathrm{~mm}$ Druck, entsprechend: $10,21 \% \mathrm{~N}$.

Für $\mathrm{C}_{15} \mathrm{H}_{14} \mathrm{~N}_{2} \mathrm{O}_{3}$ berechnet: $10,33 \% \mathrm{~N}$.

Die Vergärungsversuche werden in folgender Weise angesetzt: Versuch 5: $500 \mathrm{~g}$ Rohrzucker, $7 \mathrm{~g}$ Chlorammonium, $13 \mathrm{~g}$ Natriumbicarbonat, in $3200 \mathrm{ccm}$ Leitungswasser gelöst und mit $150 \mathrm{~g}$ frischer \&Getreidehefe gleichmäßig vermischt. Das Ganze wurde in zwei gleiche Hälften geteilt.

Die eine Hälfte a) mit einer Losung von $7 \mathrm{~g}$ reiner $\mathrm{p}-\mathrm{Oxy}-$ phenylbrenztraubensäure in $1000 \mathrm{~g}$ Wasser versetzt.

Die andere Hälfte b) mit $1000 \mathrm{~g}$ destilliertem Wasser.

säureabspaltung zu erklären; in der Tat scheint sich die Substanz schon beim Umkrystallisieren $z u$ verändern, denn es entstehen dabei aus reinen Präparaten wieder in Knollen und Kugeln von feinen Nadeln krystallisierende Stoffe.

Substanz B muß den beschriebenen Eigenschaften entsprechend als ein Kondensationsprodukt von 2 Molekülen p-0xyphenylbrenztraubensäure unter Austritt von 1 Molekül Wasser aufgefaßt werden, das noci beide Carboxylgruppen und wenigstens eine Phenolgruppe frei enthält, während die Ketongruppen offenbar die Bindung vermitteln.

Beim Erhitzen der Substanz B auf 200-220 im Vakuum entsteht unter $\mathrm{CO}_{2}$-Abspaltung eine neutrale Substanz von ganz anderen Eigenschaften. Die Untersuchung dieser Stoffe ist noch nicht abgeschlossen.

K. Fromherz. 
Versuch 6: 1000 g Rohrzucker, 15 g Chlorammonium, 26 g Natriumbicarbonat, in $4500 \mathrm{ccm}$ Wasser mit einer Aufschwemmung von $300 \mathrm{~g}$ frischer \&Getreidehefe in $1000 \mathrm{ccm}$ Wasser gleichmäßig gemischt und in zwei Hälften geteilt.

Hälfte a) mit einer Lösung von $15,2 \mathrm{~g}$ p-Oxyphenylbrenztraubensäure in $1000 \mathrm{ccm}$ Brunnenwasser versetzt.

Hälfte b) mit $1000 \mathrm{ccm}$ Leitungswasser.

Nach jeweils 2 Tagen war die Vergärung beendet; eine abfiltrierte Probe des Reaktionsproduktes gab mit salzsaurem Phenylhydrazin noch einen flockigen gelben Niederschlag (unveränderte Ketonsäure), nach $\mathrm{Ab}$ filtrieren der Fällung, Versetzen mit essigsaurem Natron und Erwärmen jedoch kein Osazon.

Die Verarbeitung des Gärproduktes erfolgte ähnlich wie bei den Versuchen mit Phenylaminoessigsäure (S. 335); die Hefe wurde abfiltriert, das Filtrat im Vakuum stark eingeengt. Versuch 5a mit $6 \mathrm{a}, 5 \mathrm{~b}$ mit $6 \mathrm{~b}$ vereinigt. Dann wurde im Extraktionsapparat mit Äther extrahiert; die Ätherextrakte wurden wiederholt mit Bisulfitlauge ausgeschüttelt, die Bisulfitlauge durch Schütteln mit frischem Äther gewaschen.

a) Hauptversuch. Die Bisulfitlösung wurde mit Salzsäure zerlegt, nach dem Verjagen der schwefligen Säure mit Äther ausgeschüttelt. Die ätherische Lösung wurde mit einer Lösung von Natriumbicarbonat durchgeschüttelt, wobei die Hauptmenge der gelösten Substanzen in die wässerige Lösung überging. (Der Äther gab beim Verdampfen nur einen geringfügigen Rückstand, der mit Eisenchlorid keine Färbung gab und nicht reduzierte, also keinen aldehydartigen Körper enthielt). Die Bicarbonatlösung enthielt unveränderte p-0xyphenylbrenztraubensäure, die durch Ansäuern, Ausäthern und Umkrystallisieren des Ätherrückstandes leicht in den charakteristischen rhombenförmigen Krystallen vom Schmelzpunkt $217^{\circ}$ gewonnen werden konnte $(3,1 \mathrm{~g})$.

Die mit Bisulfit ausgeschüttelten Ätherlösungen wurden zweimal mit frischer Natriumbicarbonatlösung gründlich extrahiert, die Bicarbonatlösung wieder mit frischem Äther; die Ätherlösungen wurden getrocknet, filtriert, der Äther abdestilliert. Der Rückstand krystallisierte sofort zu einer festen Krystallmasse. Menge:5,5 g. Durch Umkrystallisieren aus Benzol 
und Ligroin wurden daraus 4,4 g p-Oxyphenyläthylalkohol vom richtigen Schmelzpunkte $92^{\circ}$ erhalten.

Analyse: $0,0923 \mathrm{~g}$ Substanz geben $0,2354 \mathrm{~g} \mathrm{CO}_{2}$ und $0,0610 \mathrm{~g} \mathrm{H}_{2} \mathrm{O}$.

Gefunden :

$$
\mathrm{C}=69,55 \%, \mathrm{H}=7,33 \% \text {. }
$$

Berechnet für $\mathrm{C}_{8} \mathrm{H}_{10} \mathrm{O}_{2}: \mathrm{C}=69,53 \%, \mathrm{H}=7,30 \%$.

Durch Benzoylieren wurde der von Ehrlich zuerst dargestellte Dibenzoyl-p-0xyphenyläthylalkohol dargestellt, der aus heißem Ligroin, worin er leicht löslich ist, umkrystallisiert wurde: feine $\mathrm{zu}$ Büscheln angeordnete weiße Nadeln vom Schmelzpunkt $112^{\circ}$.

Aus den sauren Bestandteilen der im Bisulfit unlöslichen Fraktion (gewonnen durch Ansäuern der Bicarbonatlösung mit Salzsäure und Extraktion mit Äther) konnte noch etwa $1 \mathrm{~g}$ p-Oxyphenylbrenztraubensäure erhalten werden. Der Rest erwies sich als optisch inaktiv, er wurde nicht weiter untersucht.

b) Leerversuche. Diejenige Fraktion der parallelen Leerversuche ( $5 \mathrm{~b}$ und $6 \mathrm{~b}$ ), welche etwa vorhandenen $\mathrm{p}$-0xyphenyläthylalkohol enthalten mußte, wurde in der gleichen Weise gewonnen, wie beim Hauptversuch. Es wurden so 0,25 g eines Sirups erhalten, der jedoch nicht zur Krystallisation $\mathrm{zu}$ bringen war. Nimmt man trotzdem an, daß er aus reinem Oxyphenyläthylalkohol bestand, dann wäre immer noch der gebildete Alkohol bei der Vergärung der p-Oxyphenylbrenztraubensäure um $4-5 \mathrm{~g}$, d. i. auf das 20 fache vermehrt. (Man vergleiche auch die oben angeführten Ausbeuten an Alkohol bei den Versuchen mit Phenylaminoessigsäure S. 332).

Ein reichlicher Übergang der Ketonsäure in den Alkohol ist damit mit Sicherheit nachgewiesen. ${ }^{1}$ )

Mit dem Übergang der p-Oxyphenylbrenztraubensäure in $p$-Oxyphenyläthylalkohol ist gezeigt, daß eine Ketonsäure bei der Vergärung in den Alkohol mit der nächst niederen Zahl von G-Atomen übergeht; damit ist eine neue wesentliche

1) Ein weiterer Versuch, der dasselbe Resultat lieferte, ist weiter unten S. 346 als Kontrollversuch bei der Untersuchung der p-0xyphenylmilchsäure angeführt (Versuch 8c). 
Stütze für die Auffassung der Ketonsäure als Zwischenprodukt bei dem Abbau der Aminosäuren durch Hefe gewonnen.

Auch die Bildung des Alkohols aus der Ketonsäure

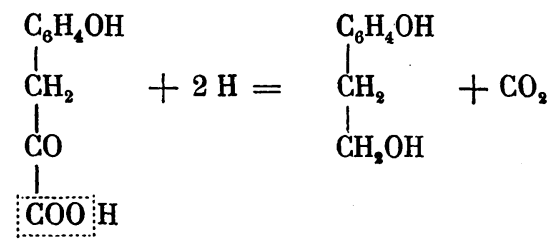

ist noch kein einfacher ProzeB; er charakterisiert sich als eine mit einer Reduktion einhergehende $\mathrm{CO}_{2}$-Abspaltung.

Auch hier ist die Entscheidung zu treffen, welcher von beiden Prozessen (Reduktion, $\mathrm{CO}_{2}$-Abspaltung) zuerst eintritt. Geht die Reduktion voran, so würde als Zwischenprodukt die entsprechende Alkoholsäure, die p-Oxyphenylmilchsäure

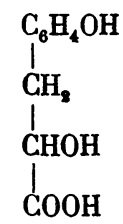

auftreten, die dann durch $\mathrm{CO}_{2}$-Abspaltung in den $\mathrm{p}$-0xyphenyläthylalkohol überginge.

Tritt dagegen zuerst die $\mathrm{CO}_{\mathbf{8}}$-Abspaltung ein, so entsteht zunächst der Aldehyd (p-0xyphenylacetaldehyd)<smiles>O=CC(O)CO</smiles>

und aus diesem durch Reduktion sekundär der p-Oxyphenyläthylalkohol. Bei der ersten Annahme wäre die bei der Vergärung der Phenylaminoessigsäure und der Phenylglyoxylsäure aufgefundene Mandelsäure (s. oben S. 334 und 336) ein Zwischenprodukt bei der Bildung des Benzylalkohols, bei der zweiten Annahme müßte man ihre Bildung als einen abnormen ProzeB, als «Parektropie, deuten.

Eine Klärung war von Gärversuchen mit der der p-0xyphenylbrenztraubensäure entsprechenden Alkoholsäure, der p0xyphenylmilchsäure, zu erwarten; ist sie das intermediäre Pro- 
dukt zwischen Ketonsäure und Alkohol, so muß sie bei der Vergärung leicht den Alkohol liefern; tut sie das nicht, so darf sie nicht als Zwischenprodukt angesehen werden.

Vergärungsversuch mit p-Oxyphenylmilchsäure. Auch hier war als Kontrolle ein Leerversuch anzusetzen; bei einem weiteren Versuch haben wir auch noch einen Parallelversuch mit Ketonsäure ausgeführt.

Versuch 7. Genau entsprechend Versuch 5 und 6 wurden $500 \mathrm{~g}$ Zucker, $7 \mathrm{~g}$ Chlorammonium, $14 \mathrm{~g}$ Natriumbicarbonat in $4000 \mathrm{ccm}$ Wasser gelöst und darin $150 \mathrm{~g}$ frische Bäckerhefe (andere, in Wiesbaden bezogene Sorte) aufgeschwemmt. Die Mischung wurde in 2 gleiche Teile geteilt, Teil b für sich vergoren, Teil a mit einer Lösung von $7,5 \mathrm{~g}$ p-0xyphenylmilchsäure (krystallwasserhaltig, Schmelzpunkt $120^{\circ}$ ), welche wir durch Reduktion der p-Oxyphenylbrenztraubensäure mit Natriumamalgam gewonnen hatten.

Nach Beendigung der Vergärung wurde abfiltriert, eingedampft, der angesäuerte Rückstand mit Äther extrahiert, die Ätherlösungen zuerst mit Bisulfit, dann mit Natriumbicarbonatlösungen ausgeschüttelt. Die dann noch im Äther gelöst bleibende Fraktion, die den p-Oxyphenyläthylalkohol enthält und bei den Versuchen $5 \mathrm{a}$ und $6 \mathrm{a}$ sofort $z u$ einer reichlichen Krystallmasse des Alkohols erstarrt war, war im Hauptversuch dem Leerversuch gegenüber nicht vermehrt und betrug in beiden Teilen $0,6 \mathrm{~g}$.

Versuch 8 . Wie oben wurden $1000 \mathrm{~g}$ Zucker, $14 \mathrm{~g}$ Chlorammonium, $26 \mathrm{~g}$ Natriumbicarbonat in Wasser gelöst, darin $250 \mathrm{~g}$ Hefe (dieselbe Sorte wie bei Versuch 7) aufgeschwemmt und auf $5000 \mathrm{ccm}$ verdünnt. Diese Mischung wurde in drei Teile geteilt:

a) Hauptversuch: $2000 \mathrm{ccm}$ der Mischung mit einer Lösung von $12,5 \mathrm{~g}$ krystallwasserhaltige $\mathrm{p}$-0xyphenylmilchsäure in $500 \mathrm{ccm}$ Wasser:

b) Leerversuch: $2000 \mathrm{ccm}$ der Mischung $+500 \mathrm{ccm}$ Wasser;

c) Parallelversuch mit $\mathrm{p}$-Oxyphenylbrenztraubensäure : $1000 \mathrm{ccm}$ der Mischung mit einer Lösung von $5,0 \mathrm{~g}$ der Ketonsäure in $250 \mathrm{~g}$ Wasser.

Nach Beendigung der Vergärung gewannen wir, wie bei den früheren Versuchen, die Ätherextrakte, die mit Bisulfit ausgeschüttelt und dann abdestilliert wurden. Der Rückstand wurde jeweils in Natronlauge aufgenommen und die stark alkalische Lösung (wie oben S. 335) mit Äther extrahiert, dann wurde mit Kohlensäure gesättigt und wieder ausgeäthert: dieser zweite Ätherextrakt, der den p-0xyphenyläthylalkohol enthält, wurde nach dem Trocknen im Vakuum über Schwefelsäure gewogen. Er betrug:

a) bei dem Versuch mit 12,5 g Oxyphenylmilchsäure 0,36 $\mathrm{g}$ (sirupös)

b), Leerversuch . . . . . . . . . 0,12, $($,

c) , Parallelversuch mit 5 g Oxyphenylbrenztraubensäure . . . . . 2,47> (Krystalle). 
Dabei ist noch $\mathrm{zu}$ beachten, daß die letzte Zahl zum Vergleich mit den bei den andern zu verdoppeln ist, da dieser Versuch mit den halben Mengen angesetzt war.

Diese Extrakte von a und b waren nicht krystallisierende Sirupe; der Extrakt $c$ dagegen erstarrte im Exsikkator alsbald zu einer Krystallmasse. Durch einmaliges Umkrystallisieren aus Benzol + Ligroin wurden 2,2 g Krystalle vom Schmelzpunkt $92^{\circ}$ erhalten.

Aus den Bicarbonatlösungen der Versuche $7 \mathrm{a}$ und $8 \mathrm{a}$ ließ sich noch ein erheblicher Teil der p-Oxyphenylmilchsäure zurückgewinnen, obwohl wegen der leichten Wasserlöslichkeit dieser Substanz an eine einigermaßen quantitative Zurückgewinnung nicht $\mathrm{zu}$ denken ist. Es wurden zusammen noch $9,0 \mathrm{~g}$ der aus Wasser umkrystallisierten Säure vom Schmelzpunkt $145-146^{\circ}$ (wasserfrei) erhalten.

Aus diesen Versuchen geht hervor, daß die p-0xyphenyl- $\alpha$-Milchsäure im Gegensatz zu der entsprechenden Ketonsäure nicht imstande ist, in nennenswerter Menge in p-Oxyphenyläthylalkohol überzugehen. Es könnten köchstens ca. $4 \%$ in den Alkohol übergegangen sein und auch das ist nicht wahrscheinlich, während von der Ketonsäure bei Versuch $8 \mathrm{c}$ über $70 \%$ in den Alkohol übergingen. Dadurch wird es höchst unwahrscheinlich, daß die Alkoholsäure als Zwischenprodukt beim Übergang der Ketonsäure oder Aminosäure in den Alkohol entsteht.

Die bei der Vergärung der Phenylaminoessigsäure entstehende 1-Mandelsäure ist also nach dem oben Ausgeführten als Produkt einer Nebenreaktion aufzufassen.

Es bleibt also nur die zweite Annahme (s. oben S. 345) daß zuerst die $\mathrm{CO}_{2}$-Abspaltung, dann erst die Reduktion eintritt, da $\mathrm{B}$ also der Weg von der Ketonsäure zum Alkohol über den Aldehyd führt.<smiles>C=CC[C@H](O)[C@H](O)CCO</smiles> 
Für diesen Weg spräche auch das Auftreten von Benzaldehyd bei der Vergärung der Phenylaminoessigsäure, ${ }^{1}$ ) wenn er nicht etwa ein Kunstprodukt ist (s. oben S. 330). Ferner ist $\mathrm{zu}$ erwarten, daß p-Oxyphenylacetaldehyd durch gärende Hefe zu p-Oxyphenyläthylalkohol reduziert wird. Diesen Versuch konnten wir bisher noch nicht ausführen; er soll nachgetragen werden, sobald wir die bis jetzt noch nicht dargestellte Substanz in genügender Menge gewonnen haben.

Wir glauben, daß unsere Versuche erlauben, den Weg, der beim Abbau der Aminosäuren durch gärende Hefe eingeschlagen wird, durch folgendes Schema wiederzugeben:

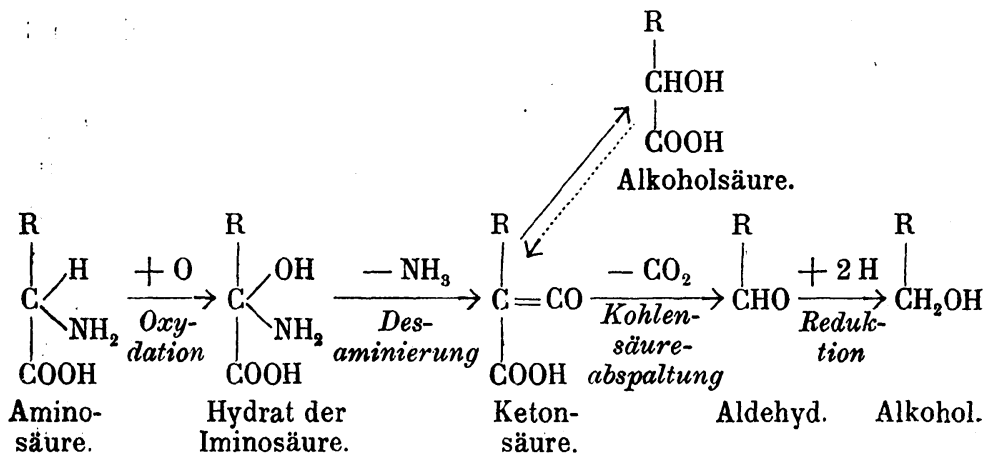

Die Acetylaminosäure, deren Entstehungsweise derzeit noch nicht geklärt ist, wurde in dieses Schema nicht aufgenommen; wir halten es zunächst für das Wahrscheinlichste, daß sie ebenso wie die Alkoholsäure als Produkt einer Nebenreaktion anzusehen ist.

Das Schema zeigt, wie der Prozeß der Bildung von Alkohol aus Aminosäure, der auf den ersten Blick als einfache hydrolytische $\mathrm{NH}_{3}$ - und $\mathrm{CO}_{2}$-Abspaltung erscheint (s. oben S.326), in Wirklichkeit viel komplizierter ist, indem bei diesen $A b$ spaltungsprozessen abwechselnde Oxydations- und Reduktionsvorgänge mitspielen.

Vergleicht man diese Art des Abbaues der Amino-

i) F. Ehrlich, Ber. d. d. chem. Ges., Bd. XL, S. 1047 (1907). 
säuren durch Hefe mit den Vorgängen beim höheren Tier, resp. in der künstlich durchbluteten Leber, so ergibt sich in beiden Fällen ein fast vollständig gleicher Verlauf. Die Bildung der Ketonsäure findet hier wie dort statt; aber auch die Annahme des Aldehyds als intermediäres Produkt bei der Umwandlung der Ketonsäure zur nächstniederen Fettsäure ist kaum zu umgehen, zumal auch hier die Entstehung der Alkoholsäure als intermediäres Produkt auszuschließen ist, denn nach den Versuchen von Neubauer und von Neubauer und Großi) liefert die Alkoholsäure des Tyrosins sowohl beim Alkaptonuriker als auch in der künstlich durchbluteten Hundeleber nicht die Abbauprodukte (Alkapton resp. Aceton), die aus der Ketonsäure entstehen.

Während aber der gebildete Aldehyd von der Hefe zum Alkohol reduziert wird, wird er vom höheren Tier zur Fettsäure oxydiert, um dann weiter verbrannt zu werden.

Die Alkoholsäure kann als Produkt einer Nebenreaktion, nämlich einer sekundären Reduktion der Ketonsäure sowohl bei der Hefegärung wie auch im höheren Tier entstehen; auch die Bildung von acetylierter Aminos äure kommt beiden Gruppen von Organismen $\mathrm{zu}$, merkwürdigerweise sind aber die so entstehenden Produkte optische Antipoden.

Ein weiterer Unterschied betrifft die beiden aromatischen Aminosäuren Phenylalanin und Tyrosin, welche beim höheren Tier insofern ein besonderes Verhalten zeigen, als auf der Stufe der Ketonsäure eine Veränderung im Benzolkern (Chinolbildung usw.) $)^{2}$ ) eintritt.

Anmerkung. Die mitgeteilten Untersuchungen über den Abbau der Aminosäuren durch Hefe scheinen auch gewisse Schlüsse auf die Vorgänge bei der Vergärung des Zuckers zu gestatten. Wenn man das oben (S. 348) aufgestellte Schema für den Abbau der Aminosäuren auf das Alanin anwendet, so ergibt sich, daß dieses über $\alpha$-Iminopropionsäure, Brenztraubensäure und Acetaldehyd in Ammoniak, Kohlensäure und Äthylalkohol übergeht:

1) O. Neubauer, D. Arch. f. klin. Med., Bd. XCV, S. 228 (1909). O. Neubauer und W. Groß, Diese Zeitschrift, Bd. LXVII, S. 228 (1910).

2) Siehe bei O. Neubauer, D. Arch. f. klin. Med., Bd. XGV, S. 246. Hoppe-Seyler's Zeitschrift f. physiol. Chemie. LXX. 


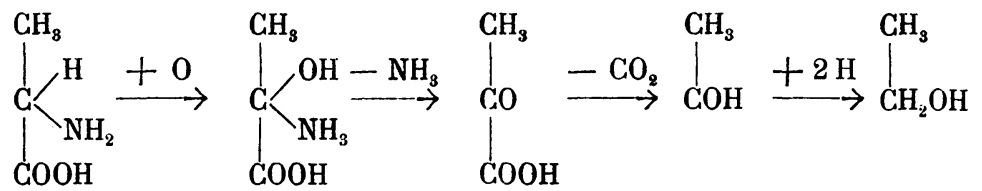

Alanin. Hydrat der a-Imino- Brenz- Acet- Äthylpropionsäure. traubensäure. aldehyd. alkohol.

Weiter ist zu schließen, daß die hier als Zwischenprodukt auftretende Brenztraubensäure durch gärende Hefe unter Reduktion $z u$ Kohlensäure und Alkohol zersetzt wird, d. h. mit anderen Worten, daß sie leicht vergärbar sein muß. Eigens angestellte Versuche, die noch nicht völlig abgeschlossen sind, haben die Richtigkeit dieses Schlusses bestätigt. Damit ist nun ohne weiteres der Gedanke gegeben, die Brenztraubensäure könnte ein Zwischenprodukt bei der alkoholischen Gärung des Zuckers sein; der Einwand, daß sie bereits eine höhere Oxydationsstufe vorstellt als der schließlich gebildete, der Alkohol, daß dementsprechend bei ihrer weiteren Zersetzung zu den Endprodukten Alkohol und Kohlensäure Energie gebunden würde, und daß es unwahrscheinlich sei, daß solche endothermischen Prozesse bei der Gärung eine Rolle spielen, ist hinfällig; denn die oben mitgeteilten Erfahrungen beim Abbau der Aminosäuren zeigen, daß derartige intermediäre Reduktionsprozesse durch gärende Hefe mit Leichtigkeit geleistet werden. Ich bitte die Fachgenossen, mir die weitere Untersuchung der Rolle der Brenztraubensäure bei der Zuckergärung zu überlassen; auch die Frage, ob sie etwa ein Zwischenprodukt bei der Zuckerverbrennung im höheren tierischen Organismus ist, soll studiert werden.

0. Neubauer. 